\title{
INFLUÊNCIA DO PH E TEMPERATURA NA ATIVIDADE ENZIMÁTICA DA ENZIMA ALFA-ACETOLACTATO DECARBOXILASE: DETERMINAÇÃO DE CONDIÇÕES ÓTIMAS UTILIZANDO PLANEJAMENTO EXEPRIMENTAL
}

\author{
J. B. M. ROCHA NETO ${ }^{1}$, A. S. PEREIRA ${ }^{1}$, D. A. B. PEREIRA ${ }^{1}$,C. M. G. F. LEMOS ${ }^{1}$, M. A. \\ LIMA $^{1}$, B. C. PESSELA ${ }^{2}$ LL. R. B. GONÇALVES ${ }^{1}$ \\ ${ }^{1}$ Universidade Federal do Ceará, Departamento de Engenharia Química \\ ${ }^{2}$ Instituto de InvestigaciónenCiencias de los Alimentos, CIAL / Consejo Superior de Investigaciones \\ Cientificas, CSIC Madrid-Espanha \\ E-mail para contato: $\operatorname{lrg} @$ ufc.br
}

\begin{abstract}
RESUMO - A enzima alfa-acetolactatodecarboxilase (ALDC) é utilizada no processo cervejeiro, sendoadicionada ao mosto inoculado com levedura para evitar a formação de diacetil, que confere sabor e aroma indesejáveis à cerveja. Neste trabalho, avaliou-se os efeitos do $\mathrm{pH}$ e temperatura sobre a atividade da enzima ALDC através de um planejamento experimental. Para tanto, utilizou-se o software Statisca 5.0 (Statsoft, USA), assegurando a validação de coeficientes dentro de um nível de confiança de $90 \%$. Um planejamento experimental fatorial completo $2^{2}$, com pontos centrais e axiais, foi conduzido e os resultados foram submetidos a análise de Pareto, análise de variância (ANOVA) e teste $F$, verificando se o modelo se ajustou adequadamente aos dados experimentais. Dentro de uma faixa de temperatura de 50 a $70^{\circ} \mathrm{C}$ e numa escala de $\mathrm{pH}$ de 4 a 10, foi observado uma influência significativa do $\mathrm{pH}$ na atividade enzimática da ALDC.
\end{abstract}

\section{INTRODUÇÃO}

As enzimas são proteínas especializadas na catálise de reações biológicas, possuindo uma extraordinária especificidade e poder catalítico, que são muito superiores aos dos catalisadores produzidos pelo homem. Entretanto, atuam em concentrações muito baixas e em condições suaves de temperatura e pH. Assim, conhecer tais condições de operação, além de controlá-las, é importantíssimo em processos industriais (VIEIRA, 2009).

A fermentação é um importante passo no processo de produção da cerveja, na qual as células de levedura usam os nutrientes encontrados no meio para acelerar o metabolismo e se multiplicar. Um importante composto carbonilo produzido na fermentação, que está presente na cerveja, é o diacetil. Este, por sua vez, acarreta ao produto uma característica organoléptica indesejável, um sabor 
amanteigado. A enzima $\alpha$-acetolactatodecarboxilase é então utilizada no processo cervejeiro, convertendo acetolactase diretamente em acetoína e, assim, evitando a formação do diacetil (HANNEMAN, 2002).

Para melhor conhecer a enzima, neste trabalho, avaliou-se a influência das variáveis temperatura e $\mathrm{pH}$ na atividade da $\alpha$-acetolactatodecarboxilase (ALDC), variáveis estas que são importantes para o controle em bioprocessos. O planejamento experimental se mostrou uma ferramenta importante, uma vez que minimiza o número de experimentos, analisando, ainda, através de ferramentas estatísticas, a validade destes. Inicialmente, determinaram-se as faixas de $\mathrm{pH}$ (4.0 a 10.0) e temperatura $\left(10^{\circ} \mathrm{C}\right.$ a $\left.50^{\circ} \mathrm{C}\right)$ que seriam utilizadas. Tais intervalos foram tomados pegando-se os extremos dos valores de $\mathrm{pH}$ e temperatura já avaliados como ótimos na metodologia utilizada como referência para medição da atividade enzimática da ALDC (STORMER, 1975).

\section{MATERIAIS E MÉTODOS}

\subsection{Determinação da Atividade Enzimática}

A atividade da enzima ALDC foi determinada através da conversão de 2acetolactato em acetoína a pH 6 e $30^{\circ} \mathrm{C}$, de acordo com a metodologia proposta por Sortmer (1975), com modificações. Uma amostra de $5 \mu \mathrm{L}$ de enzima, em $95 \mu \mathrm{L}$ de tampão MES $50 \mathrm{mM}$, foi adicionada a um eppendorf contendo $200 \mu \mathrm{L}$ de solução de substrato. Uma unidade (U) de ALDC foi definida como sendo a quantidade de enzima para liberar $1 \mu \mathrm{mol}$ de acetoína/min nas condições analisadas.

\subsection{Avaliação da influência do pH e da temperatura na atividade de ALDC}

Para se avaliar a influência do $\mathrm{pH}$ e da temperatura na atividade da enzima ALDC, utilizou-se um planejamento experimental fatorial $2^{2}$ composto central rotacional com três pontos centrais (RODRIGUES; IEMMA, 2009). A Tabela 1 mostra a matriz experimental e os resultados foram analisados com auxílio do software Statistica 5.0.

Tabela 1 - Matriz experimental e variáveis resposta do planejamento utilizado

\begin{tabular}{|c|c|c|}
\hline Ensaio & $\mathbf{p H}$ & Temperatura \\
\hline $\mathbf{1}$ & 2,75 & 30 \\
\hline $\mathbf{2}$ & 4 & 10 \\
\hline $\mathbf{3}$ & 4 & 50 \\
\hline $\mathbf{4}$ & 7 & 2 \\
\hline $\mathbf{5}$ & 7 & 30 \\
\hline
\end{tabular}




\begin{tabular}{|c|c|c|}
\hline $\mathbf{6}$ & 7 & 30 \\
\hline $\mathbf{7}$ & 7 & 30 \\
\hline $\mathbf{8}$ & 7 & 58 \\
\hline $\mathbf{9}$ & 10 & 10 \\
\hline $\mathbf{1 0}$ & 10 & 50 \\
\hline $\mathbf{1 1}$ & 11,24 & 30 \\
\hline
\end{tabular}

Os ensaios, reproduzidos inicialmente em eppendorfs de 1,5 $\mathrm{mL}$, foram conduzidos na temperatura e pH requeridos. Utilizou-se tampão MES $50 \mathrm{mM}$ no valor desejado de $\mathrm{pH}$. A temperatura foi mantida constante ao longo do tempo utilizando-se um banho termostástico durante vinte minutos. Transcorrido o tempo, o volume de cada eppendorf foi transferido para um tubo do tipo falcon, onde $4,5 \mathrm{~mL}$ de reagente de cor, constituído essencialmente de Naftol e Creatina, foram adicionados. Seguiu-se de leitura em espectrofotômetro, baseado no método colorimétrico, uma vez que a acetoína adquire coloração rosa em contato com o citado reagente de cor.

A atividade, em $\mu$ mol de acetoína/min, é então calculada como segue na Equação 2, de posse da concentração de acetoína determinada pela curva de calibração da enzima na Equação 1.

$y_{(\text {Absorbância })}=2,2685 * 10^{-2} \cdot x_{(\text {acetoínamM })}$

Unidades $/ m L$ enzima $=\underline{(\text { umol/L acetoína produzida })(5,9 \mathrm{~mL})(\mathrm{DF})}$

$$
(20 \mathrm{~min})(0,2 \mathrm{~mL})
$$

Onde:

DF é o fator de diluição utilizado na leitura;

$5.9 \mathrm{~mL}$ é o volume total da amostra;

20 minutos é o tempo de reação;

0,2 mL é o volume da solução de enzima utilizada. 


\section{RESULTADOS E DISCUSSÕES}

O planejamento experimental foi realizado com o objetivo de se determinar as condições mais adequadas à produção de acetoína. A Tabela 2 apresenta o planejamento experimental e os resultados obtidos para o estudo da influência da temperatura e do $\mathrm{pH}$ na atividade enzimática da ALDC.

Tabela 2 - Matriz experimental e variáveis resposta do planejamento utilizado

\begin{tabular}{|c|c|c|c|}
\hline Ensaio & $\mathrm{pH}$ & Temperatura $\left({ }^{\circ} \mathrm{C}\right)$ & $\begin{array}{c}\text { Atividade Enzimática } \\
(\mathrm{U} / \mathrm{mL})\end{array}$ \\
\hline 1 & 2,75 & 30 & 259,04 \\
\hline 2 & 4 & 10 & 229,52 \\
\hline 3 & 4 & 50 & 257,48 \\
\hline 4 & 7 & 2 & 4,75 \\
\hline 5 & 7 & 30 & 17,69 \\
\hline 6 & 7 & 30 & 22,63 \\
\hline 7 & 7 & 30 & 20,94 \\
\hline 8 & 7 & 58 & 37,78 \\
\hline 9 & 10 & 10 & 3,19 \\
\hline 10 & 10 & 50 & 27,76 \\
\hline 11 & 11,24 & 30 & 9,88 \\
\hline
\end{tabular}

A análise de variância (ANOVA) é apresentada na Tabela 2, onde o valor calculado de F para a atividade enzimática da ALDC foi de 16,56, sendo maior que o valor de $F_{5,5}(3.45)$ tabelado para um intervalo de confiança de $90 \%$. Assim, o modelo se afirma estatisticamente significativo, de acordo com o teste F (RODRIGUES ; LEMMA, 2009).

Tabela 2 - Análise de variância (ANOVA)

\begin{tabular}{|ccccc|}
\hline Fonte de variação & Soma quadrática & Graus de liberdade & Média quadrática & F \\
Regressão & 110866,7 & 5 & 22173,34 & 16,56 \\
Residual & 6691,5 & 5 & 1338,3 & \\
Total & 117558,2 & 10 & & \\
\hline
\end{tabular}

$\mathrm{O} \mathrm{pH}$ e a temperatura, dito ótimos, foram determinados através da metodologia de análise de superfície de resposta (BARROS NETO; SCARMINO; BRUNS, 2002). Os resultados de atividade enzimática mostrados na Tabela 2 foram utilizados para se obter os gráficos de Pareto e de superfície de resposta, conforme pode ser visto nas Figura 1 e Figura 2. 
De acordo com o Diagrama de Pareto, exibido na Figura 1, verificou-se apenas a influência do $\mathrm{pH}$ na atividade enzimática, estando, assim, além da linha de significância. Vale ressaltar que tanto o efeito linear, como o quadrático, ambos da variável $\mathrm{pH}$, tiveram efeito significativo. Enquanto isso, variações na temperatura não promoveram alterações significativas na atividade da enzima, estando abaixo da linha de significância. Ainda que combinada com o pH, a temperatura não apresentou influência relevante.

Isso nos leva a perceber que a ALDC atinge taxas de atividade relevantes e/ou próximas em diferentes temperaturas. O maior esforço seria concentrado, então, em oferecer o $\mathrm{pH}$ dito como ideal para a enzima, já que este é determinante para a atividade da enzima.

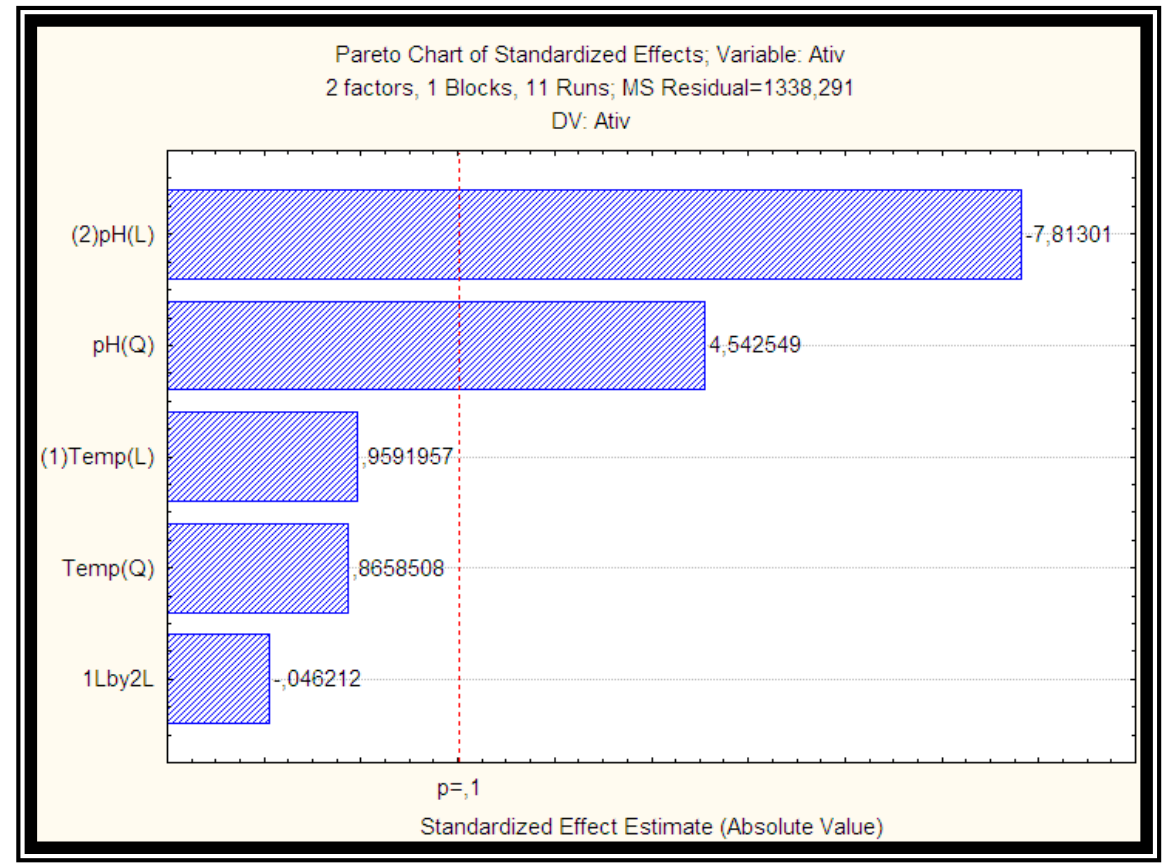

Figura 1 - Diagrama de Pareto.

A partir dos coeficientes de regressão obtidos, num coeficiente de correlação próximo de 0,95 , um modelo estatístico, para um intervalo de $90 \%$ de confiança, foi construído pelo software Statistica 5.0, relacionando a atividade enzimática da ALDC com as variáveis codificadas, $\mathrm{pH}$ e temperatura, de acordo com a Equação 3:

$$
Z=645,33-1,28 * x+0,033 * x^{2}-142,03 * y+7,77 * y^{2}-0,014 * x * y
$$

Onde: 


$$
\begin{aligned}
& \mathrm{Z} \text { - Atividade enzimática } \\
& \mathrm{x}-\mathrm{ph} \\
& \mathrm{y}-\text { Temperatura }\left({ }^{\circ} \mathrm{C}\right)
\end{aligned}
$$

Ainda através do software Statistica 5.0, um gráfico de superfície de resposta foi obtido, de acordo com a Figura 2. Através da análise deste, é possível perceber o mesmo comportamento da temperatura e do $\mathrm{pH}$, já observados no Diagrama de Pareto, na Figura 1.

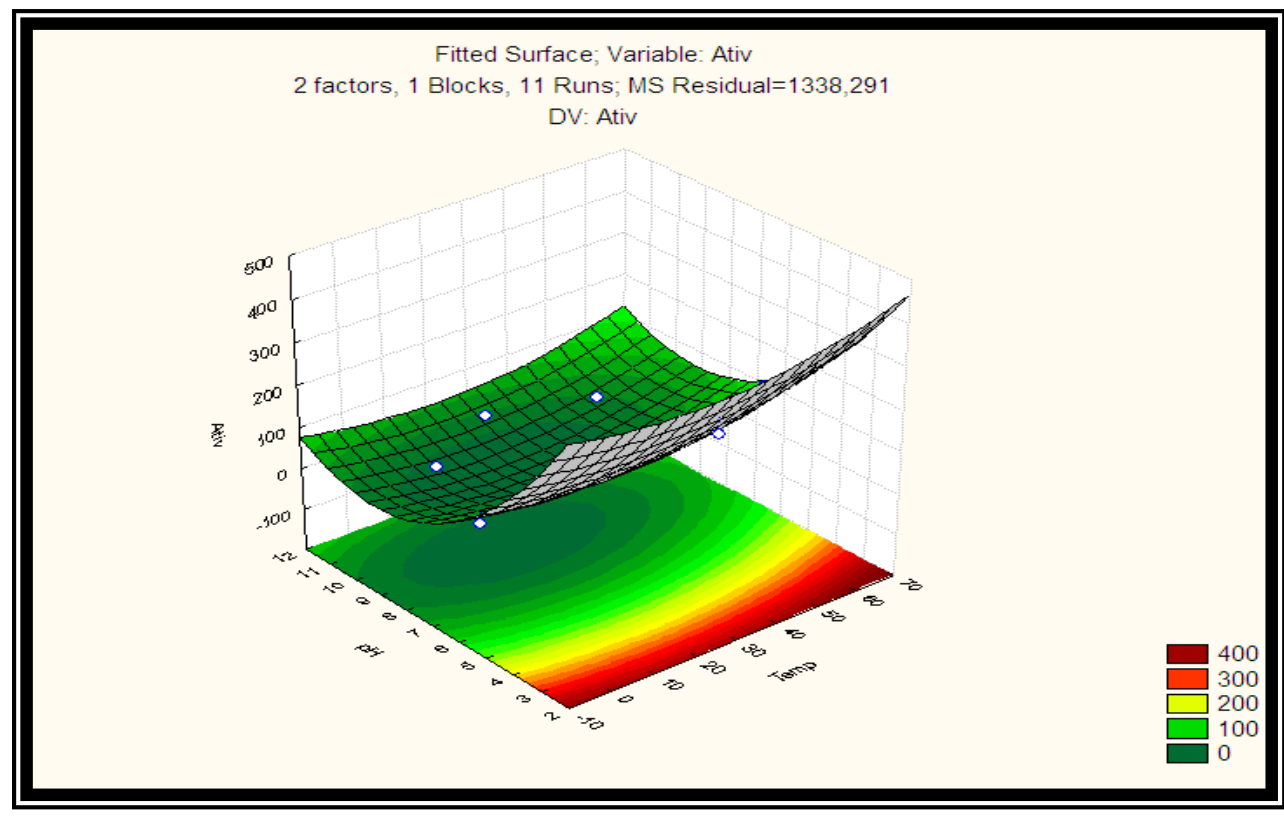

Figura 2 - Superfície de resposta para a atividade enzimática da ALDC.

As curvas de nível evidenciam uma variação muito pequena da atividade com a mudança da faixa de temperatura projetada, de $-10^{\circ} \mathrm{C}$ a $70^{\circ} \mathrm{C}$. No entanto, pode-se apontar uma tendência para $\mathrm{o}$ uso de valores mais altos de temperatura, acima de $50^{\circ} \mathrm{C}$, já que estes renderam valores um pouco maiores de atividade enzimática. Vale apontar que o estudo foi baseado nas corridas geradas para uma escala de temperatura de $10^{\circ} \mathrm{C}$ a $50^{\circ} \mathrm{C}$, gerando a já comentada projeção.

Por sua vez, o pH novamente se revela como uma variável importante, através do gráfico de superfície de resposta, uma vez que sua diminuição apresenta efeito positivo nos valores de atividade, 
em tons mais escuros na Figura 2. Portanto, trabalhar em faixa ácida com valores de $\mathrm{pH}$ menores do que 3,0, em geral, é uma forma de assegurar uma boa conversão de 2-acetolactato em acetoína.

De acordo com o gráfico de superfície de resposta na Figura 2, pode-se afirmar que a condição experimental que maximiza a atividade enzimática foi encontrada em $\mathrm{pH}<3,0$, combinada com a temperatura acima de $50^{\circ} \mathrm{C}$. A realização de um maior número de ensaios talvez melhore o ajuste dos modelos mostrados. No entanto, o estudo realizado, com coeficiente de correlação próximo de 0,95 e validado pelo teste $\mathrm{F}$, apresentou o potencial hidrogeniônico como principal e única variável independente. Portanto, ajustar o pH do tampão MES é certamente bem mais importante do que controlar a temperatura do meio reacional, uma vez que esta não altera significativamente a atividade enzimática.

\section{REFERÊNCIAS}

BARROS NETO, B.; SCARMINIO, I. S.; BRUNS, R. E. Planejamento e Otimização de Experimentos. Campinas: Editora da UNICAMP, 350p. , 2002.

HANNEMAN, W. Reducing beer maturation time while retaining quality, MBAA TQ, 39(3), pp. 149-155., 2002.

RODRIGUES, M. I.; LEMMA, A. F. Planejamento de Experimentos e Otimização de processos. Campinas, São Paulo: Editora Cárita, 2009.

STORMER, F.C. Methods in Enzymology, 1975.

VIEIRA, D. C. Imobilização da Enzima $\beta$-galactosidase de Kluyveromycesfragilis em agarose $e$ quitosana utilizando diferentes protocolos de ativação, 96f. Dissertação (Mestrado em Engenharia Química) - Departamento de Engenharia Química, Universidade Federal de São Carlos, São Carlos, 2009. 